provide a quick lead into similarly silenced loci. Also, how does this expansive epigenetic modification fit in with the ordered genetic events such as APC, K-Ras and p53 mutations during colorectal tumorigenesis? The functional consequence of 2 q14.2 silencing also remains to be defined. Finally, is there one critical gene target accompanied by a host of innocent bystanders, as is thought to be true for large deletions, or is the coordinate silencing of all of the genes important? One might also reconsider the classic examples of epigenetic tumor suppressor gene silencing, from RB and VHL to MLH1 and others. Examination of the surrounding genes will determine whether the simple paradigm of 'one methylated CpG island equals one silent gene' is in need of revision in light of this challenge from Frigola and colleagues.

1. Frigola, J. et al. Nat. Genet. 38, 540-549 (2006).

2. Baylin, S. \& Bestor, T.H. Cancer Cell 1, 299-305 (2002).

3. Jones, P.A. Semin. Hematol. 42, S3-8 (2005).

4. Feinberg, A.P. \& Tycko, B. Nat. Rev. Cancer 4, 143153 (2004).

5. Holm, T.M. et al. Cancer Cell 8, 275-285 (2005).
6. Laird, P.W. et al. Cell 81, 197-205 (1995).

7. Nephew, K.P. \& Huang, T.H. Cancer Lett. 190, 125 133 (2003).

8. Song, J.Z., Stirzaker, C., Harrison, J., Melki, J.R. \& Clark, S.J. Oncogene 21, 1048-1061 (2002).

9. Fraga, M.F. et al. Nat. Genet. 37, 391-400 (2005).

10. Akama, T.O. et al. Cancer Res. 57, 3294-3299 (1997).

11. Zardo, G. et al. Nat. Genet. 32, 453-458 (2002).

12. Hong, C., Bollen, M.I.A. \& Costello, J.F. Cancer Res. 63, 7600-7605 (2003).

13. Gonzalgo, M.L. et al. Cancer Res. 58, 1245-1252 (1998).

14. Palmisano, W.A. et al. Cancer Res. 63, 4620-4625 (2003).

15. Bernstein, B.E. et al. Cell 120, 169-181 (2005).

\title{
An adaptive path through jungle DNA
}

\author{
Juliette de Meaux
}

\section{It has long been suspected that phenotypic diversity results, in large part, from variation in DNA regulatory sequences. A new study now shows that adaptive changes in an upstream regulatory sequence of $t b 1$ are responsible for major phenotypic changes associated with maize domestication.}

Addressing the role and evolutionary importance of DNA stretches with no coding function, abundant repetitive sequences and erratic conservation patterns presents an overwhelming conceptual and technical challenge. Wholegenome arrays have supplied evidence that, beyond protein-coding DNA, a large part of the genome is transcribed ${ }^{1,2}$. Conserved stretches of noncoding DNA (ncDNA) are found throughout genomes and are likely to reflect functional regulatory features ${ }^{3-5}$. Furthermore, lack of conservation in ncDNA does not necessarily imply absence of function. Indeed, a study of the evolutionary dynamics of ncDNA in Drosophila species over a large number of genes has indicated that nonconserved nucleotide positions can participate in adaptive evolution ${ }^{6}$. On page 594 of this issue ${ }^{7}$, the idea of junk or second-class DNA is rebuked in the latest chapter of an ongoing masterpiece by John Doebley and his collaborators. Here, Richard Clark and colleagues ${ }^{7}$ report one of the most convincing examples to date of the adaptive evolutionary role of functional cis-regulatory elements buried in repetitive features. In the process, they further elucidate the genetic basis of a major phenotypic change essential to the domestication of a staple crop plant, maize.

\section{An enhancer in maize domestication}

Crosses between maize and its ancestral relative, teosinte, have revealed a small number of

Juliette de Meaux is in the Department of Genetics and Plant Breeding, Max Planck Institute of Plant Breeding Research, 50829 Cologne, Germany. e-mail:demeaux@mpiz-koeln.mpg.de quantitative trait loci (QTLs) that explain their spectacular phenotypic differences. The maize mutant $t b 1$ mimics a number of these phenotypic differences and carries an allele at one of these QTLs (reviewed in ref. 8). Notably, the $t b 1$ region exhibits an unusual pattern of diversity in maize and teosinte ${ }^{9}$. Whereas levels of diversity in the coding portion are roughly comparable to those found in teosinte, diversity in the upstream noncoding region is significantly depleted. This result is supported by the most robust test of neutrality: the Hudson-KreitmanAguadé (HKA) test. Thus, the coding region of tb1 evolved neutrally during the domestication process of maize, whereas its upstream region was subjected to strong selection. This result is consistent with the observation that changes in $t b 1$ expression could act in development to substantially alter maize architecture ${ }^{10}$. However, it remained unclear whether selection acting on a neighboring locus could have influenced diversity at $t b 1$.

Extensive sequence sampling along a 160-kb region had localized the signature of the selective sweep to a region approximately 60-90 $\mathrm{kb}$ upstream of $t b 1$ (ref. 11). This region presented an analytical nightmare best described as 'jungle DNA': it contained no predicted gene and was infested with repetitive elements. In a supreme effort, Clark et al. ${ }^{7}$ bravely characterized over 40,000 meiotic events to isolate recombinant lines between $t b 1$ and its closest neighbor. This impressive effort was necessary to fine-map the actual tb1 QTL to an $11 \mathrm{~kb}-$ wide region, approximately $60 \mathrm{~kb}$ upstream of $t b 1$. Concomitantly, an allele-specific assay of $t b 1$ expression elegantly determined that the QTL functions as a distant cis-regulatory enhancer of $t b 1$ expression. Reconstructing the $t b 1$ story has thus required an array of strategies and approaches, ranging from molecular biology to QTL mapping and population genetics, and has yielded a kernel of adaptive significance hidden in a jungle of DNA repeats.

In the past few years, maize domestication has become a heuristic model from which we learn how strong directional selection shapes the phenotype and its underlying genetic and genomic basis. Simple mutations affecting the expression or function of regulatory proteins provide the genetic basis for adaptive innovations at major QTLs for domestication phenotypes ${ }^{7,12}$. Genome scans along with efficient demographic models have estimated that $2-4 \%$ of maize genes are under selection during domestication and improvement ${ }^{13}$, and specific samplings of diversity have enabled further differentiation of genes involved in different steps of the history of maize ${ }^{14}$.

\section{Cis changes and evolutionary innovation}

Regulatory DNA has long been suspected to underlie a substantial amount of observed phenotypic variation ${ }^{15}$. Rewiring genetic networks through cis-regulatory changes should indeed permit both the generation of phenotypic novelty and the preservation of essential functions. The region delimited by Clark et al. provides a spectacular example of how changes in cis regulation can cause dramatic phenotypic changes and provide key evolutionary innovations. This discovery also reveals a major challenge facing modern biologists. Despite its depletion in 
obvious functional traits, intergenic DNA is likely to contain the genetic basis of numerous, if not most, adaptive changes. The task is daunting, in large part because the evolutionary rules governing nucleotide changes in functional noncoding DNA are not yet understood.

Several basic questions still need to be addressed. Where are the functional cis-regulatory regions? How and where are functional constraints applied in these regions? How do epistasis and pleiotropy influence the evolutionary trajectory of cis-regulatory variants? As in the case of $t b 1$ in maize, understanding the role of regulatory DNA in adaptive evolution will require a combination of approaches. Array-based high-throughput methods combined with innovative bioinformatic algorithms promise to identify key functional regulatory features in noncoding DNA. Additionally, the slow and cumbersome in-depth dissection of variation in specific adaptive traits and their underlying genetic network will have to be carried out. Indeed, only the joint analysis of historical, molecular and physiological aspects of cis-regulatory variation can reconstruct the evolutionary fate of cis-regulatory variants and elucidate their role in adaptive evolution. In this endeavor, plants promise to bring especially valuable insights because the fine-tuning of their development is environmentally defined and hence likely to meet changing requirements over evolutionary time.

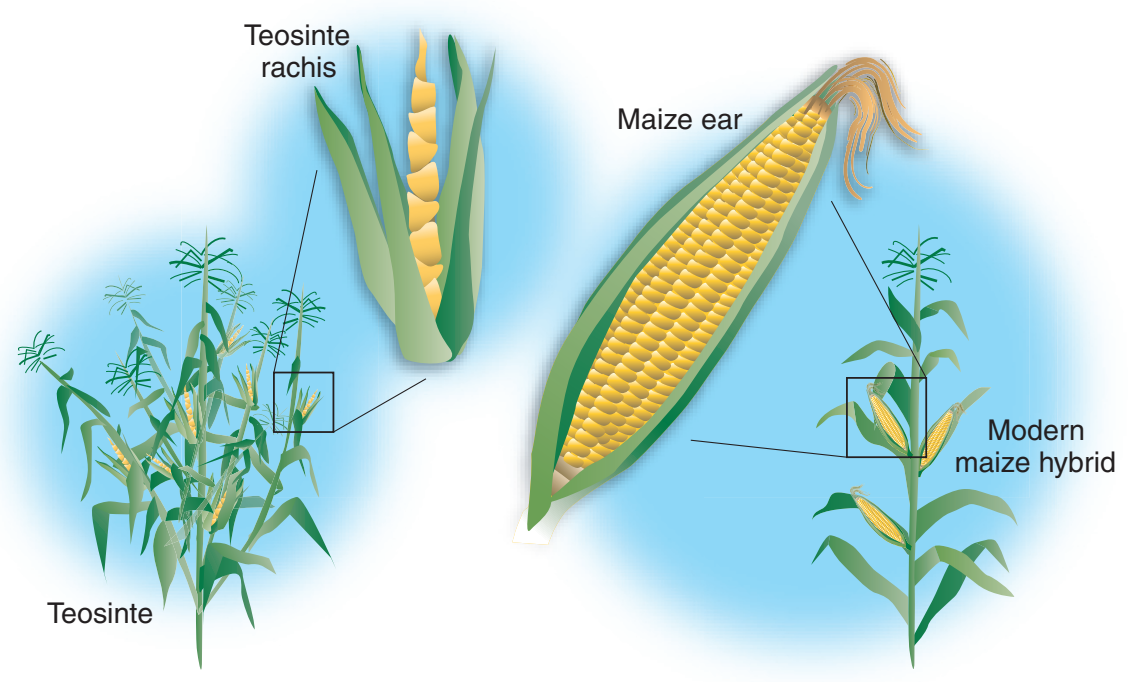

Figure 1 A substantial portion of the phenotypic differences between maize and teosinte is explained by variation in an enhancer of gene expression at the $t b 1$ locus.

1. The FANTOM Consortium. Science 309, 1559-1563 (2005).

2. Stolc, V. et al. Proc. Natl. Acad. Sci. USA 102, 44534458 (2005).

3. Willingham, A.T. et al. Science 309, 1570-1573 (2005).

4. Woolfe, A. et al. PloS Biol. 3, 116-130 (2005).

5. Washietl, S., Hofacker, I.L., Lukasser, M., Huttenhofer, A. \& Stadler, P.F. Nat. Biotech. 23, 1383-1390 (2005).

6. Andolfatto, P. Nature 437, 1149-1152 (2005).

7. Clark, R.M., Nussbaum Wagler, T., Quijada, P. \& Doebley, J. Nat. Genet. 38, 594-597 (2006).
8. Doebley, J. Ann. Rev. Genet. 38, 37-59 (2004).

9. Wang, R.L., Stec, A., Hey, J., Lukens, L. \& Doebley, J. Nature 398, 236-239 (1999).

10. Hubbard, L., McSteen, P., Doebley, J. \& Hake, S. Genetics 162, 1927-1935 (2002).

11. Clark, R.M., Linton, E., Messing, J. \& Doebley, J.F. Proc Natl. Acad. Sci. USA 101, 700-707 (2004).

12. Wang, H. et al. Nature 436, 714-719 (2005).

13. Wright, S.I. et al. Science 308, 1310-1314 (2005).

14. Yamasaki, M. et al. Plant Cell 17, 2859-2872 (2005).

15. King, M.C. \& Wilson, A.C. Science 188, 107-116 (1975).

\section{mtDNA clock runs out for dopaminergic neurons}

\section{Giovanni Manfredi}

\section{New studies indicate that damage to the mitochondrial genome (mtDNA) accumulates with age, specifically in the dopaminergic neurons of the substantia nigra implicated in Parkinson disease. These findings suggest that mtDNA damage is important in the decay of dopaminergic neurons in aging and in Parkinson disease, resulting in loss of mitochondrial function and, ultimately, neuronal death.}

mtDNA mutations and mitochondrial dysfunction have long been implicated in aging, Parkinson disease and other age-related neurodegenerative diseases. However, despite extensive efforts and a body of supporting literature, conclusive evidence has not been produced. On pages 518 and 515 of this issue, Kraytsberg and colleagues ${ }^{1}$ and Bender and colleagues ${ }^{2}$

Giovanni Manfredi is in the Department of Neurology and Neuroscience, Weill Medical College of Cornell University, New York, New York, USA. e-mail:gim2004@med.cornell.edu investigate the role of mtDNA mutations in the degeneration of dopaminergic neurons of the substantia nigra, which occurs in aging and Parkinson disease (Fig. 1). Their studies reveal accumulation of mtDNA deletions in aged dopaminergic neurons, providing evidence for the involvement of mtDNA damage in the demise of these cells.

\section{mtDNA mutations}

Mitochondria of eukaryotic cells are endowed with their own mtDNA. In humans, the mtDNA codes for a small number of proteins, 13 in total-all crucial components of the elec- tron transfer chain. In addition, the human mtDNA contains transfer RNA (tRNA) and ribosomal RNA (rRNA) genes necessary for protein translation. The genetics of mtDNA follows unique rules: mtDNA inheritance is maternal, there are thousands of copies in each cell, genes are intronless and all the genetic information is tightly packed in a small $16.5-\mathrm{kb}$ circular molecule ${ }^{3}$. mtDNA mutations associated with disease can be either maternally inherited or acquired. The former are widespread in most tissues, whereas the latter arise throughout life and remain confined to the cells in which they originated. There are 